\title{
Printed dipole antennas on substrates reinforced with unidirectional conducting fiber
}

\author{
David V. Thiel and Manimaran Kanesan \\ Griffith School of Engineering, Griffith University \\ Nathan, Queensland, Australia \\ d.thiel@griffith.edu.au
}

\begin{abstract}
Fiber reinforced composites offer strength and light weight but are electrically anisotropic. The resonant frequency of dipole antennas is orientation dependent. While FEM modeling showed a cosine relationship between the effective permittivity and angle, experimental measurements at UHF on cardboard reinforced with fine copper wire did not fit this relationship. The electromagnetic anisotropy has implications for radio communications systems located on UAVs and other platforms. Carbon nanotubes in polymeric composites provide a renewable option for high strength materials.
\end{abstract}

\section{INTRODUCTION}

Sustainable engineering practice in the field of antennas and propagation is becoming increasingly important - both during manufacture and end-of-life disposal [1]. The use of screen printed antennas and circuits embedded in plastic have been used to address these issues, particularly through the use of recycled plastic and bioplastics [2,3]. Such materials lack strength, and the inclusion of fiber into the plastic can greatly improve its strength [4]. The orientation of the fibers in the plastic during injection molding creates an anisotropic material, and thus, there can be a significant effect on the performance of an antenna [5]. Using a straight, centre-fed dipole printed on a thin anisotropic substrate, the change in the resonant frequency was used to indicate the variation in the effective length of the dipole and so the effective permittivity was deduced. This has implications for the orientation of printed antennas on fiber reinforced, shaped structures fabricated to achieve maximum strength in a predefined direction.

The thickness and electrical permittivity of the substrate can change the resonant frequency of a straight printed dipole [6]. These changes can be accommodated in the design through various approximations [7] although there is no clear analytical method to predict the effective permittivity of the substrate [8]. This follows on from the work of Galehdar et al [9] who used unidirectional and quasi-isotropic carbon impregnated epoxy to form patch and slot antennas. While carbon reinforced substrates are not particularly environmentally benign, this paper demonstrates the effects of a uni-axial substrate on dipole orientation.

\section{THEORY}

The effective permittivity $\varepsilon_{\text {eff }}$ of a thin substrate can be determined experimentally and numerically from the resonant frequency of a straight, center fed, printed dipole antenna. The effective permittivity of an anisotropic material can be determined using a rotation of the resistivity tensor in the two principal axes [10] which is different to the rotation of the conductivity tensor $[10,11]$. However, this relatively simple theory is only applicable to plane waves. The near field of a planar antenna will yield significantly different relationships for the effective permittivity as determined from the resonant frequency.

\section{NUMERICAL MODELLING}

A commercially available FEM code [12] was used to evaluate the S11 of a $158.3 \mathrm{~mm}$ long (width $4 \mathrm{~mm}$ ), center-fed, planar dipole antenna located on a thin $(t=1.6 \mathrm{~mm})$ uniaxial substrate. The substrate conductivity in the principle conducting direction (i.e. parallel to the carbon fibers) was 10 $\mathrm{S} / \mathrm{m}$. The other two axes $x$ and $z$ were assigned a conductivity of $10^{-5} \mathrm{~S} / \mathrm{m}$ and $10^{-3} \mathrm{~S} / \mathrm{m}$. The relative permittivity of the plastic was 4.5 . The dipole was aligned in several different directions $\theta$, measured with respect to the axis of maximum conductivity (minimum resistivity). The resonance frequency values were normalized to the free space resonant frequency and the effective relative permittivity calculated.

The data were fitted to a trigonometric function (equation 1) to calculate the effective permittivity $\varepsilon_{e f f}$ as a function of the $\theta$.

$$
\varepsilon_{e f f}(\theta)=A \cos ^{n} \theta+B
$$

where $n$ is the angular power law and $A$ and $B$ are constants. If $\varepsilon_{p}$ is the effective permittivity in the direction of the principal axis $\left(\theta=90^{\circ}\right)$ and $\varepsilon_{m}$ is the effective permittivity in the direction of the minor axis $\left(\theta=0^{\circ}\right)$, then (1) can be written as:

$$
\varepsilon_{e f f}(\theta)=\left(\varepsilon_{m}-\varepsilon_{p}\right) \cos \theta+\varepsilon_{p}
$$


This equation was used to estimate the effective permittivity at different orientation angles once the principal and minor axes have been measured. As this is a near field effect, the plane wave theory of [10] is not applicable.

\section{EXPERIMENTAL RESULTS}

Ribbed cardboard with $t=4 \mathrm{~mm}$, commonly available as packaging material, was reinforced with thin parallel copper wire spaced $10 \mathrm{~mm}$ apart. A $95 \mathrm{~mm}$ long straight dipole was formed from adhesive copper strip, and placed on the upper surface of the cardboard. The dipole was center-fed and the S11 was measured as a function of orientation angle $\theta$. The effective permittivity was calculated from the resonant frequency (see Fig. 1) and compared to equation (2).

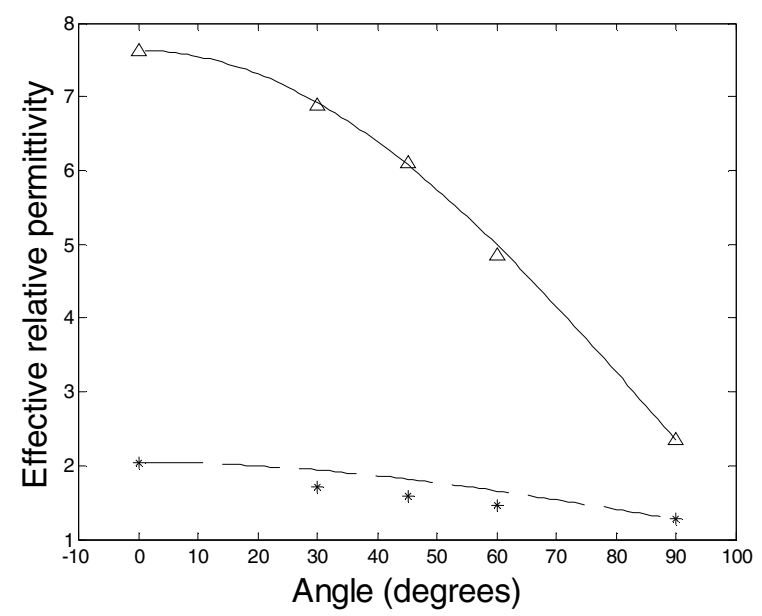

Fig. 1. Effective permittivity of a resonant dipole antenna as a function of angle on an anisotropic substrate: FEM model (triangles) and measured values *. Equation 2 was fitted to the points $\theta=0$ and $\theta=90$ and the lines are shown.

\section{DISCUSSION AND CONCLUSIONS}

The relationship between the effective permittivity $\varepsilon_{\text {eff }}$ and angle $\theta$ for a thin anisotropic, substrate is adequately described by a cosine function (equation 2) for the FEM data. However, equation (2) fails to adequately describe the experimental data although the trend remains. This is thought to be the result of the isotropic cardboard layer immediately between the dipole antenna and the anisotropic substrate formed with the copper wire.
When multiple layers of conducting fibres are included in the substrate and several different orientations, the preddiction of the effective permittivity becomes more complex.

An anisotropic substrate presents interesting challenges and perhaps some opportunities for size reduction in meander-line antennas. Fibre reinforced substrates are strong, light-weight, materials with a reduced environmental footprint. The performance of RFID antennas on fibre reinforced packaging materials will be dependent on the placement and orientation of the antenna when the reinforcing fibres are conducting and the orientation is not uniform. There is no simple theory that can be used to predict this variation in effective permittivity.

\section{REFERENCES}

[1] D.V. Thiel, "Sustainable electronics: wireless systems with minimal environmental impact," $8^{\text {th }}$ ISAPE Kunming, China, pp 1298-1301, November 2008.

[2] M. Neeli, S. Raj and D.V. Thiel, "Plastic circuit reliability and design for recycling," $11^{\text {th }}$ EPTC, Taipai, Taiwan, pp. 858-862, Dec. 2009.

[3] M. Neeli, M. Okine and D.V. Thiel, "Lifecycle analysis of plastics in South-East Queensland: recycled plastics versus bioplastics in electronics," Nanotech, vol. 3, pp. 761-764, 2013.

[4] J.N. Coleman, U. Khan and Y.K. Gun'ko, "Mechanical reinforcements of polymers using carbon nanotubes," Advanced Materials, vol. 18, pp. 689-706, 2006.

[5] L. Yang, F. Lui, H. Xia, X. Qian, K. Shen, and J. Zhang, "Improving the electrical conductivitiy of a carbon nanotube/polypropylene composite by vibration during injection moulding," Carbon, vol. 49, pp. 32743283, 2011.

[6] M. Kanesan, D.V. Thiel and S.G. O'Keefe, "The effect of lossy dielectric objects on a UHF RFID meander-line antenna," IEEE APS/URSI Symposium, July 2012.

[7] M. Kanesan, D.V. Thiel, A. Galehdar and S.G. O'Keefe, "Rapid analysis and optimization of planar Yagi-Uda antennas printed on a dielectric substrate," Int. J. RF \& Microwave CAE, DOI: 10.1002/mmce.20747, May 2013.

[8] A. Abbosh, "Accurate effective permittivity calculation of a printed centre-fed dioples and its application to quari-Yagi-Uda antennas," IEEE Trans. Antennas \& Prop., vol. 61 (4), pp. 2297-2300, April 2013.

[9] A. Galehdar, W.S.T. Rowe, K. Ghorbani, P.J. Callus, S. John and C.H. Wang, "The effect of ply orientation on the performance of antennas in or on carbon fiber composites," PIERS vol. 116, pp. 123-126, 2011.

[10] G.A. Wilson and D.V. Thiel, "Conductivity and resistivity tensor rotation for surface impedance modeling of an anisotropic half-space," Radio Sci., vol. 36 (6), pp. 1-6, Dec. 2002.

[11] N. Athanasopoulos and V. Kostopoulos, "Prediction and experimental validation of the electrical conductivity of dry carbon fibre unidirectional layers", Composites: Part B, vol. 42 pp. 1578-1587, 2011.

[12] CST Microwave Studio, www.cst.com (accessed December 2013). 